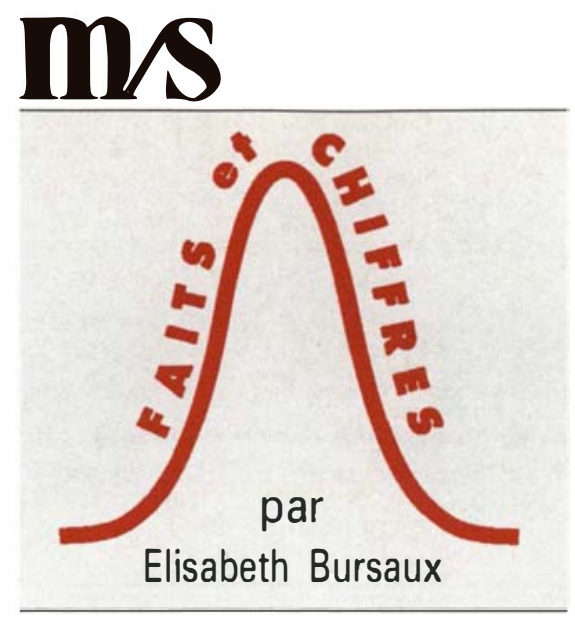

\title{
L'unification linguistique de la France
}

L 'unification linguistique de la France se poursuit malgré la diversité des langues introduites par l'immigration et les tentatives de relance des langues régionales. L'enquête menée en 1992 par l'INED et l'INSEE montre que tout se joue dans la génération qui a des enfants d'âge scolaire. A la question: "En quelle langue ou dialecte vos parents vous parlaient-ils habituellement quand vous étiez enfant? ", $16 \%$ des personnes interrogées répondent autre chose que le français. Leur demande-t-on ensuite : «En quelle langue ou dialecte parlez-vous habituellement à vos enfants? ", le chiffre tombe à $5 \%$. Ainsi, les deux tiers des parents à qui l'on parlait dans l'enfance une langue régionale ou étrangère autre que le français déclarent ne plus la parler ordinairement à leurs propres enfants.

\section{L'écrasante domination du français}

Une chute de cette ampleur en une génération (en gros depuis le milieu des années soixante) en dit long sur le pouvoir d'absorption du français. Dans $95 \%$ des familles, c'est dans la langue nationale que les parents s'adressent habituellement aux enfants. Chiffre minimum, dans lequel ne sont pas incluses les familles qui mettent une autre langue d'usage sur le même plan que le français. Comme le souligne Claude Hagège, «les langues minoritaires ne constituent pas un danger pour le français » [1]. Ainsi l'arabe est-il la première langue étrangère actuellement parlée en France entre parents et enfants, mais il concerne moins de $2 \%$ des familles, y compris celles qui déclarent l'usage simultané de l'arabe et du français.
Sa situation en France est sans commune mesure avec celle dont jouit le français au Maghreb. Le portugais, en voie de réduction rapide, ne s'emploie que dans $1 \%$ des familles. Vient ensuite l'alsacien (dialecte alémanique auquel on peut adjoindre le mosellan, dialecte francique de Metz très influent à Strasbourg). C'est de loin le premier dialecte de France : $0,6 \%$ du total national, mais entre $20 \%$ et $25 \%$ des familles d'Alsace-Moselle le parlent. Enfin, en raison d'un taux de transmission encore très élevé qui compense la faiblesse numérique d'une communauté récente, le turc réussit à devancer l'espagnol $(0,4 \%$ contre $0,2 \%)$.

$\mathrm{Si}$ l'on calcule l'ampleur de la nontransmission en une génération (Tableau I), un continuum se dessine entre, d'un côté, l'effondrement complet $(-95 \%)$ des parlers galloromans, qu'ils se rattachent à la langue d'oil ou à la langue d'oc, tous exposés de longue date à la modernisation, et, de l'autre, les pertes encore faibles $(-5 \%)$ de la langue tard venue qu'est le turc. Les autres langues s'étagent entre ces extrêmes en fonction de leur distance au français standard, qui peut être temporelle, spatiale ou sociale. Le plus souvent, ces trois dimensions se combinent.

Prenons l'exemple de la langue arabe, déjà délaissée par la moitié des parents en une génération. Voilà, pour une langue identique, des courants migratoires étalés dans le temps : en moyenne, le père et la mère se sont respectivement installés en France entre 1964 et 1972 pour les Algériens, entre 1972 et 1977 pour les Tunisiens, entre 1972 et 1978 pour les Marocains. Or l'on retrouve la même séquence dans les
Source : Héran F. Population et Sociétés, décembre 1993, $n^{\circ} 245$. 
Tableau I

TRANSMISSION DE LA LANGUE PAR LES PARENTS ÉLEVÉS

DANS UNE AUTRE LANGUE QUE LE FRANÇAIS

\begin{tabular}{|c|c|c|c|c|c|}
\hline & \multicolumn{2}{|c|}{$\begin{array}{c}\text { Langue dans laquelle ont été } \\
\text { élevés } \\
\text { les parents }\end{array}$} & \multicolumn{2}{|c|}{$\begin{array}{c}\text { Langue qu'ils parlent } \\
\text { d'habitude à leurs propres } \\
\text { enfants }\end{array}$} & \multirow{2}{*}{$\begin{array}{c}\text { Taux } \\
\text { de perte } \\
\%\end{array}$} \\
\hline & $n^{*}$ & $\%$ & $n^{*}$ & $\%$ & \\
\hline 0 . Français & & & 1415 & 68,0 & \\
\hline $\begin{array}{l}\text { 1. Parlers gallo-romans } \\
\text { 2. Créoles des DOM et de }\end{array}$ & 157 & 7,5 & 11 & 0,5 & -95 \\
\hline I'île Maurice & 75 & 3,5 & 7 & 0,5 & -90 \\
\hline 3. Italien (y compris dialectes & & & & & \\
\hline régionaux) & 181 & 8,5 & 17 & 1,0 & -90 \\
\hline dont corse & 26 & 1,0 & 3 & & -90 \\
\hline 4. Espagnol & 167 & 8,0 & 30 & 1,5 & -80 \\
\hline 5. Portuguais & 292 & 14,0 & 127 & 6,0 & -55 \\
\hline $\begin{array}{l}\text { 6. Langues non romanes } \\
\text { de France }\end{array}$ & 292 & 14,0 & 79 & 4,0 & -75 \\
\hline dont alsacien & 230 & 11,0 & 79 & 4,0 & -65 \\
\hline breton & 62 & 3,0 & & & -100 \\
\hline 7. Langues germaniques & & & & & \\
\hline et scandinaves & 100 & 5,0 & 23 & 1,0 & -75 \\
\hline ques & 90 & 4,5 & 27 & 1,5 & -70 \\
\hline dont polonais & 51 & 2,5 & 12 & 0,5 & -75 \\
\hline 9. Arabe & 439 & 21,0 & 218 & 10,5 & -50 \\
\hline 10. Langues berbères & 69 & 3,5 & 21 & 1,0 & -70 \\
\hline 11. Turc & 51 & 2,5 & 49 & 2,5 & -5 \\
\hline 12. Vietnamien & 30 & 1,5 & 14 & 0,5 & -55 \\
\hline 13. Langues d'Afrique noire & 53 & 2,5 & 13 & 0,5 & -75 \\
\hline $\begin{array}{l}\text { Autres } \\
\text { Total }\end{array}$ & $\begin{array}{r}88 \\
2085\end{array}$ & $\begin{array}{c}4,0 \\
100,0\end{array}$ & $\begin{array}{c}34 \\
2085\end{array}$ & $\begin{array}{c}1,5 \\
100,0\end{array}$ & -60 \\
\hline
\end{tabular}

* Effectifs en milliers de locuteurs; pourcentages arrondis au demi-point.

Source: enquête Éducation INSEE-INED, 1992.

Champ: hommes et femmes vivant en France avec au moins un enfant scolarisé, et dont la langue maternelle principale n'est pas le français.

Précisions sur les langues citées: 1: occitan, franco-provençal, dialectes d'oil; 2 : créoles des Antilles, de la Réunion, de l'lle Maurice; 7: anglais, allemand, néerlandais, danois...; 10: kabyle, chleuh, dialectes berbères; 13: groupe mande: madingue, soninke (ou sarakole), malinke, bambara, diula...; groupe peul: pullar, wolof, serer... ; groupe bantou: swahili, comorien, lingala, bassa, duola, diverses langues du Cameroun.

taux d'abandon de la langue: $65 \%$, $60 \%$ et $30 \%$ respectivement, même si la chute s'accélère chez les immigrés tunisiens, plus marqués par la francophonie. Il a suffi d'une génération pour que les immigrés arabophones d'Algérie se mettent habituellement à parler français en famille au même degré que les dialectophones alsaciens!

La prise en compte des écarts de temps permet également de rétablir la position exacte des deux sexes. En apparence, les femmes immigrées conservent mieux la langue maternelle que les hommes : $53 \%$ de perte contre $60 \%$. Mais ce n'est là qu'un effet de structure, leur arrivée en France étant plus tardive de cinq ans. A durée de séjour égale, et quel que soit le groupe linguistique, les femmes changent plus vite de comportement que les hommes, comme il est classique dans les situations d'exode (figure 1). Elles contribuent ainsi à réduire les écarts entre groupes. Leur conversion au français fait de la langue "maternelle » une langue plutôt paternelle, avant que celle-ci ne décline à son tour.

\section{Aux frontières du français}

La distance physique ne suffit pas à prémunir une langue contre l'absorption. L'alsacien, le breton et le corse ont beau occuper tous trois des régions périphériques, leurs situations diffèrent. Alors que la Corse reste isolée de la péninsule italique et que la Bretagne est sans arrières, l'Alsace et la Moselle s'adossent au monde rhénan. D'où le sursis accordé à l'alsacien : $40 \%$ des familles de la région le parlaient il y a une génération, $25 \%$ aujour- 


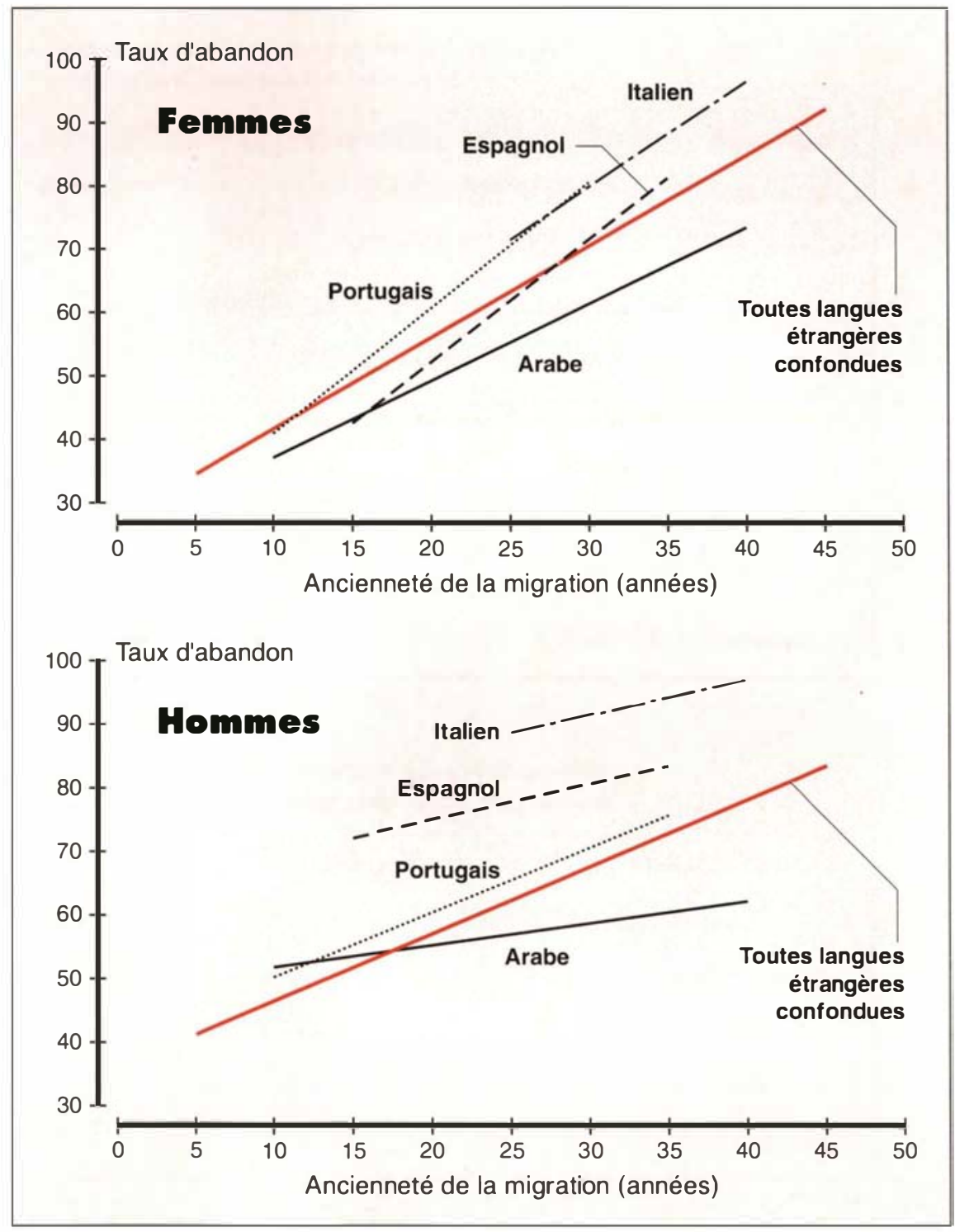

d'hui. Moins de $10 \%$ des enfants scolarisés de Corse pratiquent le corse en famille, au point qu'il pourrait être devancé dans l'île par l'arabe et l'italien. Quant au breton, le groupe social qui le cite le plus volontiers comme langue d'enfance est celui des instituteurs, signe qu'il est aujourd'hui bien éteint comme langue familiale ordinaire: des 44 parents de l'échantillon qui le pratiquaient jeunes, aucun ne l'a transmis. Une étude régionale de l'INSEE confirme la quasi-disparition du breton chez les moins de trente ans.

$\mathrm{m} / \mathrm{s} n^{\circ} 3$ vol. 10, man 94
Autre exemple d'une distance physique réduite par la durée du contact culturel, le cas des langues créoles ou des parlers occitans, abandonnés neuf fois sur dix. Les locuteurs entérinent la domination du français en parlant spontanément de "patois" à l'enquêteur, et, qui plus est, d'un patois départemental ("corrézien ", " ariégeois », « aveyronnais », etc.), selon une définition bien républicaine du local.

Le cas des langues d'Afrique noire est plus complexe. Est-ce parce que les locuteurs sont exposés de longue
Figure 1. Proportion de parents qui renoncent à parler habituellement leur langue d'origine aux enfants chez les familles immigrées. Données exprimées en tendances linéaires. Source: enquête Éducation, INSEE-INED, 1992.

date à la francophonie ou qu'ils sont traditionnellement rompus au multilinguisme? Toujours est-il qu'elles sont délaissées trois fois sur quatre, c'est-à-dire pas moins que l'anglais ou l'allemand chez les immigrés venus de pays limitrophes.

\section{La richesse du pauvre}

A la distance physique s'ajoute ou se substitue la distance sociale. Tous langues et dialectes confondus, la part de non-francophone dans la relation parents-enfants ne cesse de 
croître à mesure que l'on descend l'échelle sociale. Quand on compare, dans un même groupe linguistique, les parents qui transmettent et ceux qui ne transmettent pas, on constate que les premiers sont toujours plus démunis socialement (figure 2). Le trésor linguistique dont ils sont dépositaires est bien une richesse, mais c'est souvent la richesse du pauvre. Ils ne peuvent en tirer parti pour assurer les autres tâches de transmission, en particulier l'aide à l'enfant dans son travail scolaire.

Plus attachés au pays d'origine et à ses traditions (religieuses notamment), les parents transmetteurs ont aussi plus souvent épousé un conjoint de même langue. Assurément, l'ancienneté de la migration et, plus encore, l'âge plus ou moins précoce à la migration contribuent à distinguer les deux groupes (six années les séparent), mais la comparaison entre immigrés et régionaux montre assez que l'œuvre du temps est loin de tout expliquer. Pour une même durée de séjour, il subsiste des différences substantielles parmi les immigrés eux-mêmes.

Toutes choses égales par ailleurs, les trois principaux facteurs qui expliquent le maintien de la langue d'une génération à l'autre sont la sortie de l'école avant 12 ans, le mariage avec un conjoint de même langue et le désir de rentrer un jour au pays. Moins important est le fait de partager avec le conjoint une pratique religieuse, signe d'homogénéité culturelle du ménage. Autant de facteurs qui peuvent retarder mais d'une génération seulement le passage au français.

\section{La famille relayée par l'école?}

Depuis 1951, la loi Deixonne autorise, sur la base du volontariat des maîtres et des familles, l'enseignement des langues régionales dans le second cycle (tout en excluant les "dialectes allogènes": alsacien, corse, flamand). A la suite de la circulaire Savary de 1982, qui étend ce principe à toutes les langues sans distinction, les instituteurs peuvent

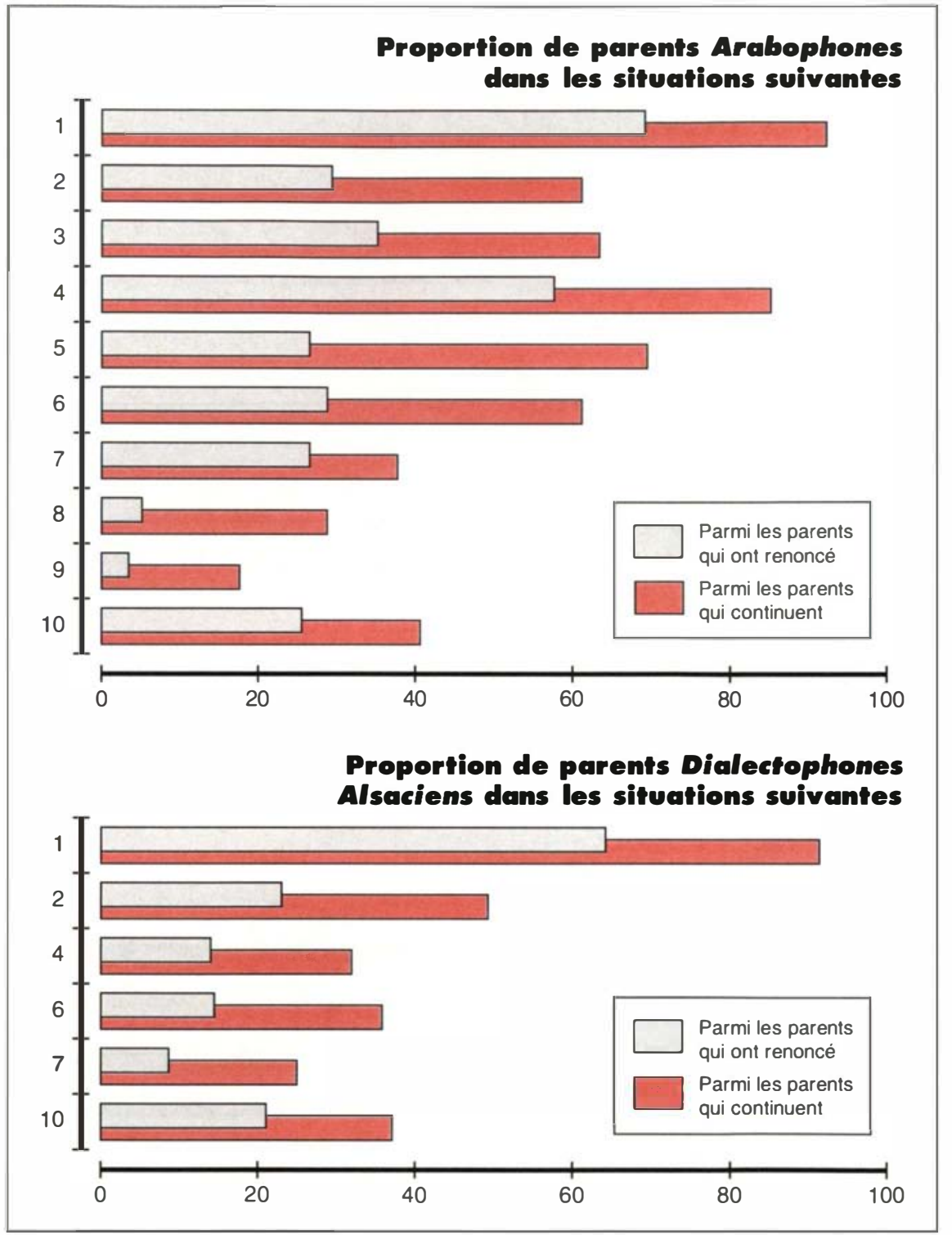

Figure 2. Situation comparée des parents, selon qu'ils continuent de parler habituellement leur langue à leurs enfants ou qu'ils $y$ renoncent.

1: a un conjoint de même langue,

2: couple ou parent isolé pratiquant une religion,

3: a quitté l'école avant 12 ans,

4: sans diplôme,

5: déclare lire difficilement le journal en français,

6 : se dit très souvent dépassé pour aider ses enfants dans le travail scolaire,

7: ouvrier non qualifié,

8: souhaite que l'enfant garde sa nationalité d'origine,

9: l'enfant a commencé sa scolarité dans le pays d'origine,

10: l'enfant a redoublé une classe.

Source: enquête Éducation, INSEE-INED, 1992. 
suivre des cours facultatifs de "langue et culture régionales " (LCR) et former à leur tour des groupes d'élèves volontaires. Mais, de l'avis des spécialistes, l'impact reste très marginal : au mieux quelques centaines d'élèves dans les régions concernées [2]. Peut-on vraiment attendre du système scolaire la revitalisation d'un patrimoine qu'il a contribué à détruire par le passé ? Régionale ou étrangère, une langue a beau retrouver sa dignité en devenant langue d'école, elle cesse d'être familière si elle n'est plus familiale. Enfin, même retransmise par une fraction des familles, une langue peut s'altérer. L'alsacien d'aujourd'hui est truffé de périphrases calquées du français et d'emprunts directs : les jeunes locuteurs ne cessent de "zapper " entre les deux langues comme ils le font entre télévision allemande et télévision française. Ia même hybridation s'observe dans la pratique du corse ou celle de l'arabe dialectal. Bref, le recul des langues régionales ou immigrées sous la pression du français semble encore plus sévère que ne le suggère la simple considération des taux de pratique.

\section{RÉFÉRENCES}

1. Hagège C. Ie franşais el les siècles. Paris: Odile Jacol), 1987.

2. Vermes (i, Boutet J. France, pays multilingue. Paris: I.'Harmattan, 1987. 